\title{
ÉDITORIAL
}

\section{Prise en charge locorégionale multidisciplinaire en cancérologie}

\author{
Y. Belkacemi ${ }^{1}$, M. Héry ${ }^{2}$ \\ ${ }^{1}$ AP-HP, CHU Henri-Mondor et Université de Paris XII, Créteil, France \& Association de radiothérapie et d'oncologie \\ de la méditerranée (Arome ; www.aromecancer.org) \\ ${ }^{2}$ Centre hospitalier Princesse-Grâce, avenue Pasteur, F-98000 Monaco, France \\ Correspondance : yazid.belkacemi@hmn.aphp.fr
}

La multidisciplinarité est devenue une règle incontournable de la pratique en cancérologie. En dehors des urgences ou des cas particuliers, il est de moins en moins concevable de poser une indication ou de définir une stratégie thérapeutique sans que cela ne soit validé lors d'une réunion de concertation pluridisciplinaire (RCP) qui doit aboutir à l'établissement d'un programme personnalisé de soins (PPS) décrivant le protocole thérapeutique décidé et communiqué au patient lors d'une consultation dédiée.

En France, la dynamique de la pluridisciplinarité généralisée s'est inscrite dans le cadre défini par la circulaire du 22 février 2005 relative à l'organisation des soins en cancérologie (mesure 32 du Plan cancer I) au sein des établissements chargés de la prise en charge des cancers. Même s'il est probablement encore tôt d'évaluer l'impact exact de cette réorganisation sur la survie par pathologie, on sait que l'amélioration de la survie est multifactorielle pour la majorité des cancers et qu'elle est rarement due au seul progrès thérapeutique. La plus grande précocité du diagnostic (du fait du dépistage) ou la simple évolution des méthodes de diagnostic sont des éléments clés à considérer. L'étude EUROCARE-4, portant sur des patients traités dans 23 pays européens (83 registres, 2,7 millions de cas de cancers inclus), fait apparaître une amélioration des taux de survie relative dans les pays occidentaux et l'amorce d'une diminution des écarts entre les pays de I'Est et ceux de l'Ouest, liée aux avancées obtenues en cancérologie [1].

À l'évidence, l'amélioration des traitements locorégionaux apparaît comme un facteur déterminant pour espérer, à l'avenir, une réduction encore plus significative de ces écarts. Ces bénéfices seront obtenus grâce à des actions à tous les niveaux, comprenant la sensibilisation et la formation de plus en plus pointue des professionnels, la promotion des guidelines spécifiques aux traitements locorégionaux et systémiques et des actions plus précoces sur le dépistage et la prévention.

Le traitement locorégional est le socle de la prise en charge des cancers localisés. Son efficacité peut conditionner, dans de nombreux cas, la survie à long terme des malades. Quelle que soit l'arme thérapeutique utilisée, la règle du traitement minimal efficace aux dépens du traitement maximal tolérable doit prévaloir au moment de poser l'indication d'un traitement. En effet, il s'agit de maintenir la balance du ratio bénéfice/risque systématiquement en faveur du bénéfice. En chirurgie, les avancées dans la réduction de l'étendue des gestes, les performances des techniques chirurgicales tumorales, ganglionnaires modernes et cœlioscopiques, l'arrivée de la chirurgie robotisée ont révolutionné la pratique. En radiothérapie, les avancées technologiques et informatiques ont permis un formidable progrès dans la définition des volumes cibles et l'épargne des tissus sains. Enfin, dans le cadre des traitements à visée de réduction tumorale, les associations thérapeutiques avec de nouvelles molécules, voire des thérapies ciblées suscitent un véritable espoir dans certaines localisations.

Ce numéro spécial, dédié au traitement locorégional, est élaboré sur la base de la transversalité et de la multidisciplinarité. II a pour objectifs de faire le point :

- sur les recommandations pour l'optimisation des traitements locaux de différents cancers en termes de qualité d'exérèse tumorale ; 
- sur la prise en charge ganglionnaire en chirurgie et en radiothérapie ;

- sur les progrès et l'apport de la robotique à la chirurgie abdominopelvienne ;

- sur les avancées de la radiothérapie de haute technicité.

Grâce à l'expertise et aux expériences des équipes sollicitées, nous espérons que ce numéro spécial sera, pour les lecteurs, l'une des références pour ce début d'année 2010.

\section{Référence}

1. Berrino F, De Angelis R, Sant M, et al. (2007) Survival for eight major cancers and all cancers combined for European adults diagnosed in 1995-1999: results of the EUROCARE-4 study. Lancet Oncol 8(9): 773-83 